\title{
An End-to-End Mechanism for Jitter Control in Multimedia Services
}

\author{
A. La Corte, A. Lombardo, S. Palazzo, G. Schembra \\ Istituto di Informatica e Telecomunicazioni, University of Catania \\ V.le A. Doria 6 - 95125 Catania - Italy \\ phone: +39 95339449 - fax: +3995338280 \\ e-mail: lacorte, alombard, palazzo, schembra@iit.unict.it
}

\begin{abstract}
In this paper, we propose an end-to-end mechanism for the achievement of synchronization requirements in multimedia retrieval services over broadband integrated networks. The synchronization requirements are specified by users in terms of delay jitter and loss probability. The mechanism is based on a feedback protocol which uses correlation properties of the arrival times of the data streams to compute the retrieval times of the next Information Units (IUs). Moreover, an architectural framework in which the users' QoS synchronization requirements and the proposed synchronization mechanism fit together is presented. This framework is used to illustrate how the synchronization mechanism parameters have to be determined in order to meet the user requirements.
\end{abstract}

\section{Keywords}

Multimedia communications, intramedia synchronization, jitter.

\section{INTRODUCTION}

The next generation of distributed applications will involve multiple media running on general purpose personal computers interconnected via integrated service networks. Such applications require both intramedia and intermedia synchronization. Intramedia synchronization defines the time relationships of a single medium over a single connection; intermedia synchronization defines those relationships for multiple media concurring into a 
multimedia data flow (Yen,1994). Maintaining synchronization among and within multimedia streams is a very critical QoS target which affects the design of the whole multimedia system. Although in the last few years various papers dealing with the problem of both synchronization requirements specification (Anderson,1991) (Campbell, 1994) (Little,1991) (Ravindram, 1993) (Steinmetz,1993) (Znati,1993) and synchronization algorithm development (Escobar, 1992) (La Corte, 1995) (Ramanathan, 1993) (Santoso, 1993) (Zarros, 1994) have been published, the design of a framework which allows users to actually require and obtain a specified QoS is a problem which is far from being solved.

In this paper we illustrate an end-to-end mechanism which can be applied to obtain a given QoS in terms of delay jitter and loss probability in multimedia retrieval services. In the proposed mechanism the arrival times are predicted at the destination site and fed back to the source site. In the latter the retrieval times are computed in order to smooth the delay jitter. At the destination site a buffer is inserted in order to further smooth delay jitter, if necessary. In order to guarantee the effectiveness of the mechanism and, on the other hand, to entail an acceptable feedback traffic overhead, a suitable figure of merit which allows us to monitor the synchronization degree at the destination site is introduced. The paper also shows how in our approach the system manager is enabled to tune the mechanism parameters to the delay QoS requirements of the multimedia application.

The rest of the paper is organized as follows. Section 2 outlines the problem of synchronization in the frame of the data location model assumed and describes an architectural framework where user requirements, the intramedia synchronization algorithms and the network fit together. Section 3 introduces the synchronization algorithm to be applied to each monomedia stream to compensate for end-to-end delay jitter, and shows how the resulting feedback traffic overhead can be reduced and adapted according to the value of a suitable figure of merit representing the observed degree of synchronization. Section 4 allows us to illustrate how the mechanism parameters can be determined in order to meet the users' QoS delay requirements. Some simulation results which show the effectiveness of the intramedia synchronization algorithm are also presented. Finally, Section 5 summarizes the paper.

\section{PROBLEM DEFINITION}

A multimedia stream, as shown in Figure 1, is the combination of $N$ monomedia streams which are retrieved from different sources and transported by the network, for example through separate virtual channels. Each monomedia stream, is seen as being made up of an ordered sequence of Information Units (IUs). At the end-to-end level, an IU is a logical atomic information unit, although it may be segmented into smaller units (packets) according to the transport mechanism used in the integrated network.

Let us consider the generic $\mathrm{i}$-th media. The $\mathrm{IU} \mathrm{U}_{\mathrm{i}}(\mathrm{n}) \mathrm{s}$ are retrieved at the source site at the instants $t_{-} t_{i}(n)$; they are subject in transmission to a variable delay, which is related to the characteristics of the channel conveying the monomedia stream, and are received at the destination site at the instants $\mathrm{t}_{-} \mathrm{rx}_{\mathrm{i}}(\mathrm{n})$. We refer to the IU latency time or IU delay as the time interval between IU retrieval at the source and IU delivery in the buffer at the destination, when it is available to be scheduled, processed and delivered to the user. 


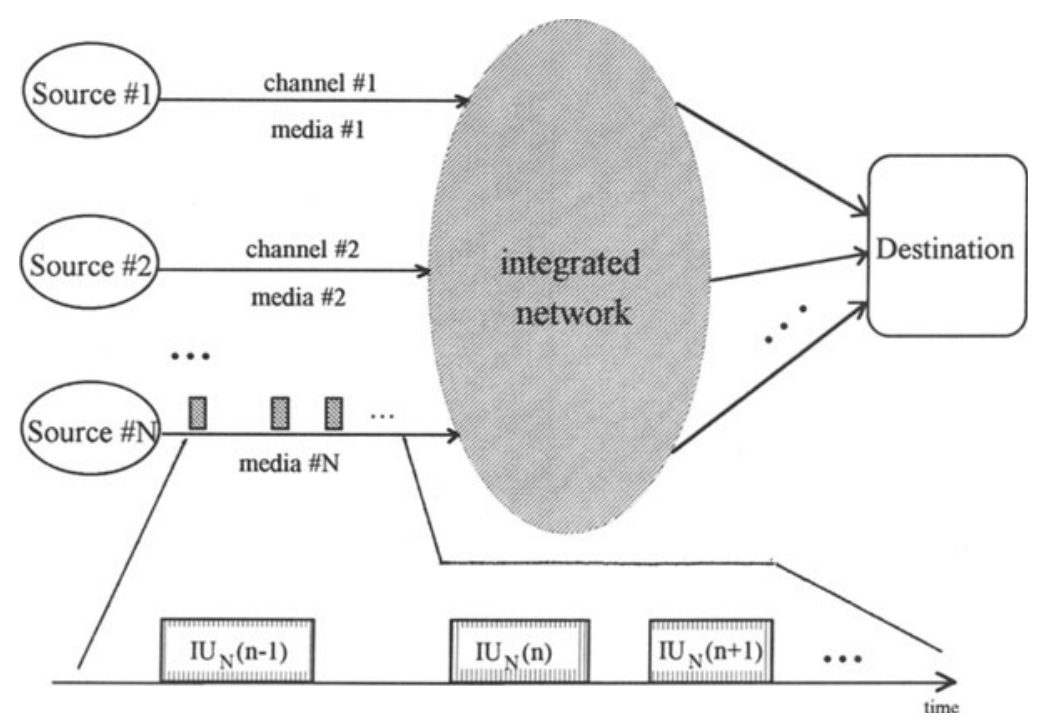

Figure 1 Monomedia streams in a multimedia retrieval service and Information Units (IUs).

We assume that clock ticks at the source sites and at the destination site have the same advancement, but the current local times may be different (Santoso,1993). Therefore the latency time of the $\mathrm{IU}_{\mathrm{i}}(\mathrm{n})-\mathrm{d}_{\mathrm{i}}(\mathrm{n})$ - is linked to retrieval times by the following relation:

$t_{-} \mathrm{rx}_{i}(\mathrm{n})=\mathrm{t}_{-} \mathrm{tx} \mathrm{x}_{i}(\mathrm{n})+\mathrm{d}_{\mathrm{i}}(\mathrm{n})$

If we denote as $\mathrm{j}_{\mathrm{i}}(\mathrm{n})$ the latency time jitter or, more simply, jitter, of the $\mathrm{n}$-th IU of the $\mathrm{i}$-th media, and as $\bar{d}_{i}$ the mean latency time of the IUs of the $\mathrm{i}$-th media, (1) becomes:

$t \_r x_{i}(n)=t \_t x_{i}(n)+\bar{d}_{i}+j_{i}(n)$

As $j_{i}(n)$ is a random process, the temporal relationships between the IUs of the $\mathrm{i}$-th media are not maintained at the destination site. Obviously, if the jitter suffered by any IU is null, the intramedia synchronization condition is achieved. The intramedia synchronization problem can thus be solved by introducing suitable algorithms for the achievement of the condition $\mathrm{j}_{\mathbf{i}}(\mathrm{n}) \equiv 0$.

In order to define a suitable measure of synchronization degree in the multimedia 
distributed system, let us consider the IU interarrival times for a single media. As shown in Figure 2 , interarrival times are linked to jitter. Therefore, we can say that the $\mathrm{i}$-th monomedia stream is synchronized if:

$\mathrm{y}_{\mathrm{i}}(\mathrm{n}) \equiv \mathrm{x}_{\mathrm{i}}(\mathrm{n}) \quad$ for any ' $\mathrm{n}$ '

where ' $x_{i}(n)$ ' is the sequence of the interarrival times of IUs from the $\mathrm{i}$-th media at the source site and ' $y_{i}(n)$ ' is the sequence of the interarrival times at the destination site. So (3) - is a condition for intramedia synchronization.

If relation (3) is satisfied, the autocorrelation sequences of the interarrival times at the source and destination site satisfy the condition

$\Delta R^{(i)}(m)=R_{y y}^{(i)}(m)-R_{x x}^{(i)}(m) \equiv 0 \quad$ for any ' $m$ '

Therefore if $\Delta \mathrm{R}^{(\mathrm{i})}(\mathrm{m}) \neq 0$, it can be assumed as a measure of lack of synchronization. The lower the values assumed by the function $\left|\Delta R^{(i)}(\mathrm{m})\right|$, the better the intramedia synchronization. Equation (4) is thus an alternative expression of the condition for intramedia synchronization.

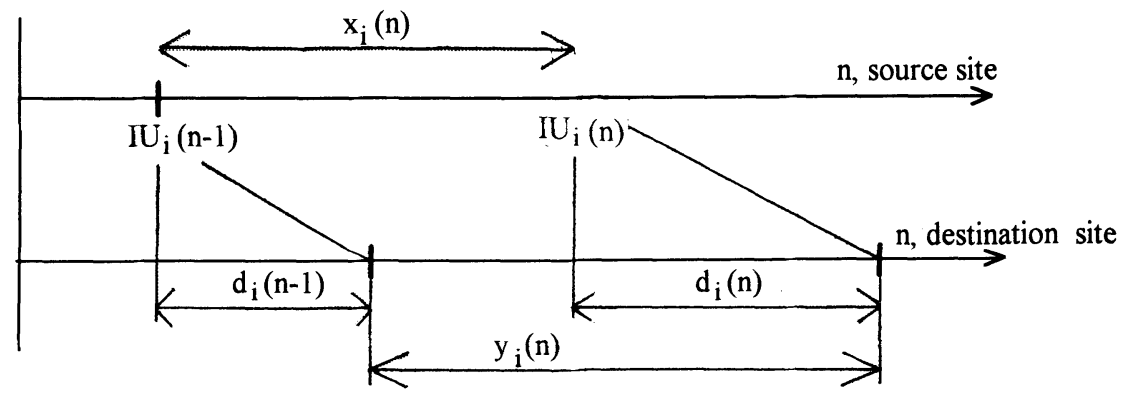

Figure 2 Interarrival times and latency times.

A simple way to achieve intramedia synchronization is to vary the IU retrieval times at the source site so as to compensate for the latency time jitter. In general, however, multimedia applications can support a jitter provided it is limited (Steinmetz,1993); therefore, as far as the synchronization property is concerned, user requirements can be expressed as follows:

$\operatorname{prob}\left[\mathrm{j}_{\mathrm{i}}(\mathrm{n}) \geq \mathbf{J}_{\mathrm{i}, \max }\right] \leq \varepsilon_{\mathrm{i}}$

if the multimedia service to be provided is loss sensitive, or 
$\left\{\begin{array}{l}\left|j_{i}(n)\right| \leq J_{i, \max } \\ \operatorname{prob}(\operatorname{loss}) \leq \varepsilon_{i}\end{array}\right.$ for any ' $n^{\prime}$

if the multimedia service to be provided is delay sensitive, assuming that the IU is lost when the jitter exceeds the maximum admissible value.

In other words, in the former case a suitable mechanism has to be designed in order to guarantee that any $\mathrm{IU}_{\mathrm{i}}(\mathrm{n})$ is delivered and no more than $\varepsilon_{\mathrm{i}} \%$ of the total amount of IUs delivered suffers a jitter greater than $\mathrm{J}_{\mathrm{i} \text {,max }}$. In the latter case all the $\mathrm{IU}_{\mathrm{i}}(\mathrm{n})$ delivered must suffer a jitter lower than $\mathrm{J}_{\mathrm{i} \text {,max }}$; IUs that suffer more than $\mathrm{J}_{\mathrm{i}, \max }$ at the destination have to be discarded; however, the percentage of discarded IUs cannot exceed $\varepsilon_{i} \%$. In this paper we take into account the loss probability due to the exceeding of time limits only, that is, the network is not considered to be lossy. If this is not the case, the probability $\varepsilon_{\mathrm{i}}$ also has to include any network loss probability other than that due to synchronization requirements. Figure 3 shows an architectural framework where user requirements, the intramedia synchronization algorithms and the network fit together.

In order to guarantee that the above QoS requirements can be achieved for each media regardless of the QoS provided by the underlying network, it is necessary to reshape the probability density function (pdf) of the jitter. This can be achieved by varying the IU retrieval time according to the mechanism introduced in the next section.

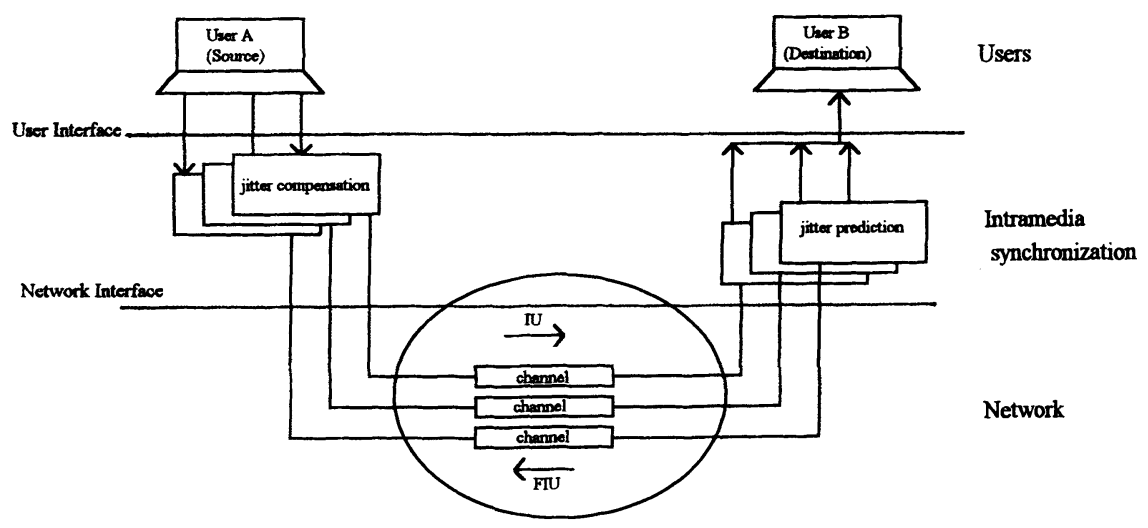

Figure 3 Architectural framework for the intramedia synchronization mechanism and user's QoS synchronization requirements. 


\section{END-TO-END JITTER COMPENSATION MECHANISM}

The end-to-end jitter compensation can be achieved by predicting the jitter and anticipating the retrieval time of the value corresponding to this predicted value. Jitter prediction can easily be computed at the destination site, so it is necessary to exchange information between the destination and source sites. The information units that the destination sends to the source for this purpose are called Feedback Information Units (FIUs), and the relative data flow is called the feedback flow.

The amount of feedback flow can be adapted to the synchronization status of each media and therefore to the delay QoS requirements of the multimedia application. If, in fact, the synchronization condition (4) is met at the destination site, besides a negligible error, there is no need for FIUs at the source, as intramedia synchronization has practically been achieved.

In the light of what has been said so far, the steps of the algorithm we propose are the following:

- at the destination site, for each IU received, calculation of $\Delta \mathrm{R}^{(\mathrm{i})}(\mathrm{m})$ and comparison with a suitable mask: if $\Delta R^{(i)}(m)$ is outside the mask the predicted jitter value is embedded in an FIU and fed back to the source;

- at each source site:

1. if an FIU is received, the retrieval time of the next IU is anticipated by the predicted jitter value contained in the FIU;

2. if no FIU is received, the predicted jitter value is calculated on the basis of the jitter values predicted previously and the retrieval time of the next IU is anticipated by this value.

This solution follows the scheme shown in Figure 4.

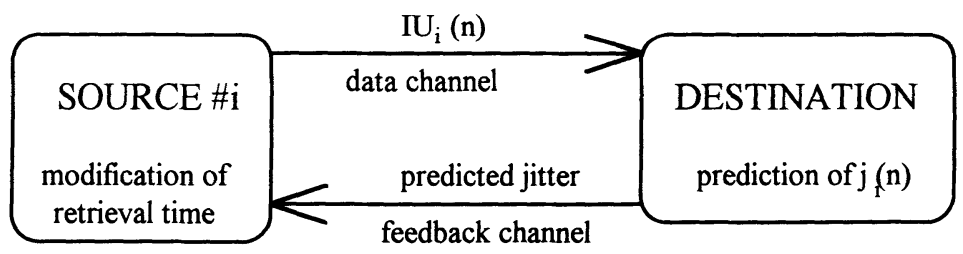

Figure 4 Prediction-based end-to-end jitter compensation scheme.

The predicted jitter value at the destination site can be calculated by optimal prediction, e.g. by means of a linear prediction, in such a way that the variance of the prediction error $e_{i}(n)$ is minimized (Therrien,1992). Note that the jitter prediction error, $e_{i}(n)$ is the jitter actually felt at the destination site after application of the jitter compensation mechanism. To achieve such a prediction the jitter values actually provided by the network, $\mathrm{j}_{\mathrm{i}}(\mathrm{n})$, must available at the destination site. This can be calculated at the destination site as 
$\mathrm{j}_{\mathrm{i}}(\mathrm{n})=\mathrm{t}_{-} \mathrm{rx}_{\mathrm{i}}(\mathrm{n})-\mathrm{t} \_\mathrm{tx}_{\mathrm{i}}(\mathrm{n})+\mathrm{j}_{\mathrm{i}}(\mathrm{n})-\overline{\mathrm{d}}_{\mathrm{i}}$

where $\hat{j}_{i}(n)$ is the predicted jitter value when the previous IU is received at the destination site, provided that timestamps are attached to each IU, the latency time is a stationary process in a broad sense, and its mean value does not depend on the statistical characteristics of the source.

When no FIU is received at the source site, prediction is less reliable than that performed at the destination site. In fact, the predicted jitter value is progressively obtained by using the jitter values previously predicted. In this case the predicted jitter value at the source site tends to zero and, therefore, the jitter prediction error gradually grows. The system is, however, stable both on account of the kind of predictor used and because, when the prediction error exceeds a certain range of values, the function $\Delta R^{(i)}(m)$ falls outside the mask of admissible values, thus reactivating transmission of FIUs at the destination site and bringing the lack of synchronization back within admissible limits. For this reason the choice of the mask for the function $\Delta \mathrm{R}^{(\mathrm{i})}(\mathrm{m})$ determines the degree of trade-off between feedback traffic overhead and prediction error.

Obviously, the effectiveness of the prediction-based mechanism depends on the statistical properties of the jitter, in particular its autocorrelation function. Any residual jitter that the mechanism presented above cannot compensate for, can be compensated for by a suitable buffer so as to guarantee the QoS requirements.

\section{QOS REQUIREMENTS CONTROL}

In order to provide the user with the QoS required at the user interface it is necessary to determine, for each media ' $\mathrm{i}$ ', how to reshape the residual jitter so that the intramedia synchronization mechanism will guarantee the QoS requested.

If an optimal predictor is used, the jitter prediction error is practically uncorrelated and has a bell-shaped distribution which can be approximated quite well with a normal distribution (Therrien,1992). In this case, the parameter which gives analytical indications of the effect of reshaping is jitter variance. QoS parameters can thus be expressed in terms of the admissible jitter variance, $\sigma_{i, a}^{2}$, which results in prob $\left[e_{i}(n) \geq J_{i, \max }\right] \leq \varepsilon_{i}$, which, in turn, on the basis of (5) or $\left(5^{\prime}\right)$ determines the QoS at the user interface.

As the distribution of the jitter prediction error is normal, by using Gitnik's approximation (Gitnik, 1991) we obtain that the admissible jitter prediction error variance of the $\mathrm{i}$-th media $\sigma_{i, \mathrm{a}}^{2}$ must satisfy the relation:

$$
\sigma_{i, a}^{2}=-0.619536 \cdot \mathrm{J}_{\mathrm{i}, \max }^{2} / \ln \left(2 \cdot \varepsilon_{\mathrm{i}}\right)
$$

To guarantee the QoS required the mean square error of the prediction error has to be lower than or equal to the variance obtained previously, i.e. 
$\sigma_{i, e}^{2}=E\left[e_{i}^{2}(n)\right] \leq \sigma_{i, a}^{2}$

So, once we have calculated the admissible jitter prediction error variance which will guarantee that the delay $\mathrm{QoS}$ requirements are met, the predictor characteristics required to meet condition (8) can be determined.

Of course, the possibility of obtaining a given jitter prediction error variance depends on the degree of correlation of the delay jitter provided by the network (Thierren,1992). Therefore, in the real case the minimum obtainable prediction error variance may be greater than the admissible jitter prediction error variance $\sigma_{i, a}^{2}$. In this case, to guarantee QoS requirements, the prediction-based jitter compensation mechanism have to be used with a compensation buffer. This buffer is inserted after the prediction-based jitter compensation mechanism. The resulting jitter pdf is of the kind shown in Figure 5. The admissible jitter prediction error variance must now satisfy the relation:

$\sigma_{i, a}^{2}=\frac{-0.619536}{\ln \left(2 \cdot \varepsilon_{i}\right)}\left(\mathrm{J}_{\mathrm{i}, \max }+\mathrm{b}_{\mathrm{i}} / 2\right)^{2}$

where $b_{i}$ is the buffer size.

The value of $b_{i}$ in (9) has to be chosen in such a way that $\sigma_{i, a}^{2}$ is greater than the minimum obtainable prediction error variance, thus making the prediction-based mechanism effective.

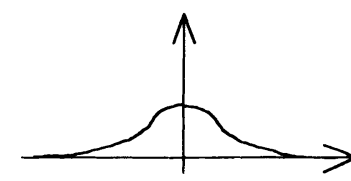

(a)

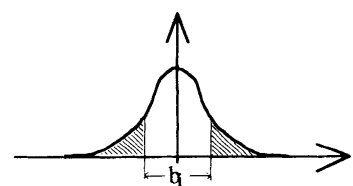

(b)

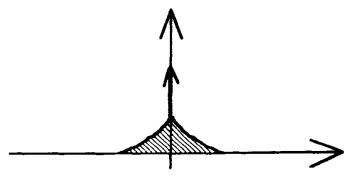

(c)

Figure 5: jitter pdf supplied by the network (a), after application of the prediction-based mechanism (b) and after the compensation buffer.

If an FIU is sent for each IU and an optimal predictor is used, the jitter prediction error is practically uncorrelated. Thanks to this property in the steady state we obtain:

$$
\left|\Delta R^{(i)}(m)\right| \cong \begin{cases}2 \cdot \sigma_{i, e}^{2} & \text { for } m=0 \\ 0 & \text { for } m \neq 0\end{cases}
$$

Therefore the figure of merit which gives analytical indications of the effect of pdf reshaping is the difference between the admissible jitter prediction error variance and twice 
the short-term jitter prediction error variance $\sigma_{i, e}^{2}(n)$. So FIUs are sent at the source site when $\left|\Delta \mathbf{R}^{(i)}(n, m)\right|_{m=0} \geq s_{t h} \cdot \sigma_{i, a}^{2}$, where $s_{t h}$ is a scale factor, by means of which it is possible to tune the trade-off between the reduction in the jitter prediction error variance and the amount of overhead traffic.

In order to gain a clearer understanding of how the parameters involved in the proposed mechanism can be determined in accordance with the aim of guaranteeing a certain QoS at the user interface, we will now discuss simulation of the proposed synchronization mechanism as applied to a video retrieval service.

Without losing in generality, we refer to a network environment with the following characteristics:

- a delay whose average value was assumed to be equal to $300 \mathrm{~ms}$;

- a latency time jitter with a normal distribution, a null average and a variance of $\sigma^{2}=100$ $\mathrm{ms}^{2}$;

- an exponential jitter autocorrelation sequence, that is, $R(m)=\sigma^{2} \cdot a^{m}$;

- a video source which every $1 / 30$ of a second sends an IU corresponding to a video frame coded with a variable bit rate technique.

We also assume that the type of predictor used for the mechanism is an 8-th order linear one. The autocorrelation sequence was attributed an exponential trend to make the longterm prediction error variance independent of the order of the predictor (Thierren,1992). The value of ' $a$ ' was set according to the level of delay jitter correlation to be represented $(a=0.9$ for a highly correlated jitter, $\mathrm{a}=0.75$ for a jitter with medium correlation, $\mathrm{a}=0.5$ for a jitter with low correlation and $\mathrm{a}=0.1$ for a practically uncorrelated jitter).

To assess the efficiency of the prediction-based mechanism, we initially established that an FIU would be sent for each IU so as to obtain the minimum jitter prediction error variance $\sigma_{e_{-} \min }^{2}$. This situation allows the best performance to be calculated, that is, the best jitter pdf reshape that the end-to-end jitter compensation mechanism can provide.

In this case the amount of information that has to be fed back obviously depends on the speed at which the IUs are received. In the case of a $2 \mathrm{Mbit} / \mathrm{s}$ videoconference service, for example, the IU may be a video frame, so transmission of the predicted value requires about one FIU every $1 / 30$ of a second. If we consider a packet of 44 octets, the number of packets needed to transmit the FIUs is on average less than $0.6 \%$ of the number of packets needed to transmit the IUs. If "finer" synchronization is desired, an IU of a smaller size can be considered (e.g. a subfield, line, etc.), with a corresponding percent increase in the bit rate of the feedback channel.

Table 1 shows the $\sigma_{e_{-} \text {min }}^{2}$ values achieved when various jitter correlation levels are considered. Of course, $\sigma_{e_{\_} \text {min }}^{2}$ grows as the jitter correlation level decreases.

Let us assume, for example, that the user QoS requirements are $\varepsilon_{\mathrm{i}}=10^{-4}, \mathrm{~J}_{\mathrm{i}, \mathrm{max}}=30 \mathrm{~ms}$. From (7) we get $\sigma_{a}^{2}=65.5 \mathrm{~ms}^{2}$. If the jitter correlation is high or medium (i.e. $\mathrm{a}=0.9$ or $\mathrm{a}=0.75$ ), from the values given in Table 1 it can easily be deduced that $\sigma_{a}^{2}>\sigma_{e_{-} \min }^{2}$. If, on the other hand, the jitter correlation is low $(\mathrm{a}=0.5)$ it is not possible to obtain $\sigma_{\mathrm{e}}^{2} \leq \sigma_{\mathrm{a}}^{2}$ without a compensation buffer. 
Table 1: Minimum jitter prediction error variance with various levels of jitter correlation in the considered simulation scenario.

\begin{tabular}{ccccc}
\hline $\mathrm{a}$ & 0.9 & 0.75 & 0.5 & 0.1 \\
\hline$\sigma_{\mathrm{e} \_\min }^{2}\left(\mathrm{~ms}^{2}\right)$ & 20 & 44 & 75 & 99 \\
\hline
\end{tabular}

Once the prediction criterion and the compensation buffer size have been fixed according to the required admissible jitter prediction error variance, by monitoring the value of $\Delta R^{(i)}(n, 0)$ it is possible to achieve a good trade-off between the reduction in the jitter prediction error and the amount of feedback traffic. Table 2 reports the jitter prediction error variance and the throughput of the feedback flow with various correlation levels and values of $\varepsilon$, when $\mathrm{J}_{\max }=30 \mathrm{~ms}$ and $\mathrm{s}_{\mathrm{th}}=1.8$. For the throughput of the feedback flow it was assumed that an ATM cell was used for each FIU. The Table also gives the admissible jitter prediction error variance values and the buffer size needed to guarantee the QoS parameters. Buffer size values of zero mean that the prediction-based mechanism can meet the QoS requirements on its own. $b$ values corresponding to a null FIU throughput mean the buffer size needed to meet the QoS requirements without using the prediction-based mechanism.

Table 2 Prediction error variance, throughput of the feedback flow and compensation buffer size $\left(\mathrm{J}_{\max }=30 \mathrm{~ms}\right.$ and $\left.\mathrm{s}_{\mathrm{th}}=1.8\right)$.

\begin{tabular}{|c|c|c|c|c|c|c|c|c|}
\hline \multirow[b]{2}{*}{$\mathrm{a}$} & \multirow[b]{2}{*}{$\begin{array}{l}\sigma_{\mathrm{e}}^{2} \\
\left(\mathrm{~ms}^{2}\right)\end{array}$} & \multirow[b]{2}{*}{$\begin{array}{l}\text { FIU } \\
\text { (cell/s) }\end{array}$} & \multirow{2}{*}{$\frac{\varepsilon=10^{-3}}{\sigma_{\mathrm{a}}^{2}}$} & \multirow[b]{2}{*}{$\begin{array}{l}\mathrm{b} \\
(\mathrm{ms})\end{array}$} & \multirow{2}{*}{$\begin{array}{l}\varepsilon=10^{-4} \\
\sigma_{\mathrm{a}}^{2} \\
\left(\mathrm{~ms}^{2}\right)\end{array}$} & \multirow[b]{2}{*}{$\begin{array}{l}\mathrm{b} \\
(\mathrm{ms})\end{array}$} & \multirow{2}{*}{$\frac{\varepsilon=10^{-5}}{\sigma_{\mathrm{a}}^{2}}\left(\frac{\left.\mathrm{ms}^{2}\right)}{\left({ }^{2}\right)}\right.$} & \multirow[b]{2}{*}{$\begin{array}{l}\mathrm{b} \\
(\mathrm{ms})\end{array}$} \\
\hline & & & & & & & & \\
\hline 0.9 & 20 & 30 & 89.7 & 0 & 65.5 & 0 & 51.5 & 0 \\
\hline 0.9 & 28 & 24 & 89.7 & 0 & 65.5 & 0 & 51.5 & 0 \\
\hline 0.9 & 32 & 18 & 89.7 & 0 & 65.5 & 0 & 51.5 & 0 \\
\hline 0.9 & 61 & 7 & 89.7 & 0 & 65.5 & 0 & 61 & 5.3 \\
\hline 0.9 & 89 & 2 & 89.7 & 0 & 89 & 10 & 89 & 18.6 \\
\hline 0.9 & 100 & 0 & 100 & 3.4 & 100 & 14.2 & 100 & 23.6 \\
\hline 0.75 & 45 & 30 & 89.7 & 0 & 65.5 & 0 & 51.5 & 0 \\
\hline 0.75 & 46 & 27 & 89.7 & 0 & 65.5 & 0 & 51.5 & 0 \\
\hline 0.75 & 47 & 18.5 & 89.7 & 0 & 65.5 & 0 & 51.5 & 0 \\
\hline 0.75 & 66 & 14 & 89.7 & 0 & 66 & 0.3 & 66 & 7.9 \\
\hline 0.75 & 91 & 3 & 89.7 & 0 & 91 & 10.8 & 91 & 19.8 \\
\hline 0.75 & 100 & 0 & 100 & 3.4 & 100 & 14.2 & 100 & 23.6 \\
\hline 0.5 & 76 & 30 & 89.7 & 0 & 76 & 4.7 & 76 & 12.9 \\
\hline 0.5 & 85 & 26.6 & 89.7 & 0 & 85 & 8.4 & 85 & 17.1 \\
\hline 0.5 & 90 & 13 & 89.7 & 0 & 90 & 10.4 & 90 & 19.3 \\
\hline 0.5 & 95 & 4.6 & 95 & 1.8 & 95 & 12.3 & 95 & 21.5 \\
\hline 0.5 & 99 & 4 & 99 & 3.1 & 99 & 13.8 & 99 & 23.2 \\
\hline 0.5 & 100 & 0 & 100 & 3.4 & 100 & 14.2 & 100 & 23.6 \\
\hline
\end{tabular}




\section{CONCLUSIONS}

In this paper a mechanism for the achievement of synchronization requirements in multimedia retrieval services has been presented, based on an adaptive feedback technique for jitter prediction. The method proposed calculates the retrieval times using some correlation properties of the latency times of the data streams. Moreover, the use of an additional compensation buffer has been investigated. Some simulation results which show the effectiveness of our approach in a case study regarding a multimedia retrieval service have also been presented.

\section{REFERENCES}

D. P. Anderson D. P., Homsy G.: "A Continuous Media I/O Server and Its Synchronization Mechanism", vol. 24, IEEE Computer, October 1991.

Campbell A., Coulson G., Hutchinson D.: "A Quality of Service Architecture", Computer Communication Review, vol 24, no. 2, April 1994.

Escobar J., Deutsch D., Partridge C.: "Flow Synchronization Protocol", Proc. Globecom '92, Orlando, December 1992.

Gitnik A. P.: "The approximation of the probability integral by elementary functions", Telecommunication and Radio Engineering, USSR, 35-36, 1991.

Yen W., I.F. Akyildiz: "On the Synchronization Mechanisms for Multimedia Integrated Services Networks", International COST 237 Workshop, Vienna, November 1994.

La Corte A., Lombardo A., Palazzo S., Schembra G.: "QoS Requirement control in delay jitter sensitive multimedia services", Proc. ICC'95, Seattle, June 1995.

Little T. D. C., Ghafoor A.: "Multimedia Synchronization Protocols for Broadband Integrated Services", IEEE Journal on Selected Areas in Communications, vol. 9, no. 9, December 1991.

Ramanathan S., Venkat Rangan P.: "Adaptive Feedback Techniques for Syncronized Multimedia Retrieval over Integrated Networks", IEEE/ACM Transaction on Networking, vol. 1, no. 2, April 1993.

Ravindram K., Bansal V.: "Delay Compensation Protocols for Synchronization of Multimedia Data Streams", IEEE Transaction on Knowledge and data engineering, vol. 5, no. 4, August 1993.

Santoso H., Dairaine L., Fdida S., Horlait E.: "Preserving Temporal Signature: a Way to Convey Time Constrained Flows", Proc. Globecom 1993, Houston, November 1993.

Steinmetz R., Engler C.: "Human perception of media synchronization", IBM European Networking Center, Technical report 43.9310.

Therrien C. W.: "Discrete Random Signals and Statistical Signal Processing", Prentice Hall, 1992.

Zarros P. N., Lee M. J., Saadawi T. N.: "Statistical Synchronization Among Participants in Real Time Multimedia Conference", Proc. INFOCOM '94, , Toronto, June 1994.

Znati T., Field B.: "A Network Level Channel Abstraction for Multimedia Communication in Real-Time Networks", IEEE Transaction on Knowledge an Data Engineering, vol. 5. no. 4, August 1993. 


\section{BIOGRAPHIES}

Aurelio La Corte received his degree in Electrical Engineering from the University of Catania, Italy, in 1988 and a Doctorate degree in Electronic and Computer Sciences in 1992. $\mathrm{He}$ is now Assistant Professor at the University of Catania. His research interests include traffic modelling and services in high-speed networks and multimedia systems.

Alfio Lombardo received a degree in Electrical Engineering from the University of Catania, Italy, in 1983, where he is now Associate Professor of Telematics. His current research interests include distributed multimedia applications, network management and broadband communications.

Giovanni Schembra received a degree in electrical engineering from the University of Catania in 1991. He is completing his studies for a Doctorate degree in Electronic and Computer Sciences. His interests include traffic analysis in broadband networks and the modelling of multimedia applications.

Sergio Palazzo received his degree in Electrical Engineering from the University of Catania, Italy, in 1977, where he is now Associate Professor of Telecommunication Networks. His research interests include broadband networks, MAN architecture and protocols, mobile network planning and multimedia applications. 